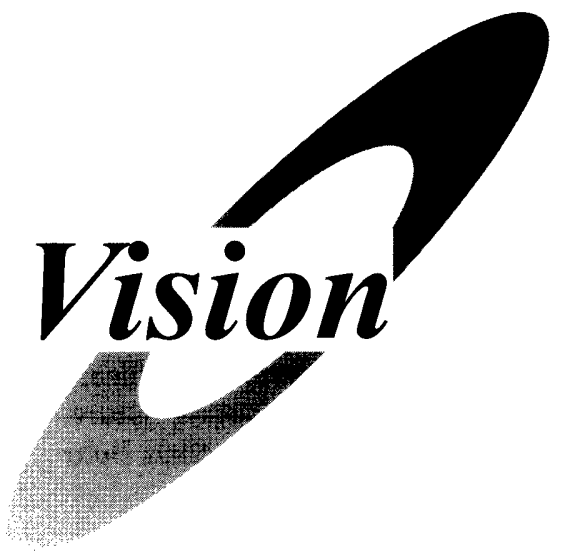

\title{
Electrochemical Sensors: Key Products for our Technological Future
}

Isao TANIGUCHI

Developing new, world-leading science and technology is essential for constructing our future. In Japan, we have a long tradition of manufacturing a wide range of materials and finely-crafted devices. Chemical sensors, the preparation of which often requires delicate manual techniques, fit our national character well, and this field of science and technology has become one of our strongest. As is well-known, chemical sensors have become a key technology in wide areas of our industry and daily life.

Of course, new sciences and technologies should act in the service of human welfare. Energy, safety, comfort, recycling, harmonic development and preservation of the environment are some of the key themes for new technologies at present and even more so in the future. Chemical sensors are required for all these fields. Many new sensors have been developed in recent years, and the list of applications continues to grow. These include detecting chemical and biological poisons and weapons, monitoring the safety and origin of chemicals, monitoring the freshness and taste of daily foods, health monitoring and mental care, and monitoring environmental conditions in the home and in the workplace. A prominent research theme at present is the development of sensors that are extremely small, sensitive, selective and intelligent, but have a simple structure. Small or imbedded sensors for medical use will also be required to discriminate between individuals. The combination of various electronic techniques and information-processing methods with sensor devices provides more sophisticated sensor systems. We should also consider ways in which other sciences such as brain science can contribute to our field. Another theme is the non-destructive detection of the desired information. As society's and industry's needs become more diverse, it is incumbent on us to analyze and anticipate these needs carefully. To bring the knowledge and insights of various fields together in a meaningful way, the exchange of ideas among scientists and engineers of different fields becomes more and more important.

In the coming years, we may expect original work with a large impact to be developed in this country. These new findings and technologies can also be expected to give birth to new industries or small businesses. To be at the frontiers of science and technology, our society as a whole, including the university, may well have to adapt, and we may need to countenance not only organizational and structural change but also changes to our ways of thinking. In such a situation, electrochemistry itself, though a science with a long history, has also been changing in significant ways.

Functional interfaces have recently become a key topic of interest. There are now various techniques by means of which control at the atomic and molecular levels (socalled nano-technology), with direct observation of the structures, is possible. Through functional interfaces, new devices are being provided for purposes such as energy conversion and information (or signal) transferring. Using well-designed functional interfaces, electrochemical sensors are becoming a key science and technology for growing new industries.

I cordially invite you to join us in expanding the field of electrochemistry and contributing to the future of our society from the perspective of world-leading science and technology.



Isao TANIGUCHI Vice president of the Society

Dean, Faculty of Engineering, Kumamoto University 